\title{
Relações de negócio em hotelaria: um modelo estrutural aplicado ao segmento empresarial
}

\author{
ARMANDO LUÍS VIEIRA ${ }^{\mathrm{a}}$, CARLOS COSTA
}

RESUMO 0 presente artigo tem como objetivo investigar a influência da importância e da qualidade das relações de negócio na decisão de uma empresa em manter o envolvimento com determinado hotel. Para tratar a questão, é proposto um modelo testado com base em dados de um inquérito realizado a uma amostra final de 483 representantes de pequenas e médias empresas (PMEs) portuguesas, que manifestaram as suas perceções sobre as relações de negócio com os seus interlocutores privilegiados numa cadeia hoteleira. Os dados foram analisados com recurso à técnica estatística de modelação em equações estruturais. 0 estudo produz vários resultados importantes. A qualidade da relação é influenciada por variáveis interpessoais, com destaque para o papel do Gestor de Clientes (i.e., o representante do hotel), e por outras associadas à relação propriamente dita. Quanto à importância da relação, vários fatores surgem como indicadores significativos, tais como os benefícios da relação e os custos da sua interrupção. Os resultados sugerem que a importância da relação assume um papel preponderante na determinação da propensão de interação futura. Em termos de contribuição teórica, a investigação acrescenta valor ao paradigma do marketing relacional, ao testar e validar um modelo da influência da importância da relação, adicionalmente aos efeitos da qualidade da relação, na propensão de interação futura entre PMEs e hotéis. 0 estudo sugere igualmente implicações práticas de gestão, com vista ao sucesso das estratégias de marketing relacional e com o objetivo da fidelização dos clientes e rentabilização das relações de negócio com eles estabelecidas.

PALAVRAS-CHAVE importância da relação, marketing relacional, modelação em equações estruturais, qualidade da relação, relações de negócio.

\section{HISTORIA DEL ARTÍCULO}

¿CÓMO CITAR?:

Vieira, A.L. \& Costa, C. (2016). Relações de negócio em hotelaria: um modelo estrutural aplicado ao segmento empresarial. Perspectiva Empresarial, 3(1), 93-107. http:// dx.doi.org/10.16967\%2Frpe.v3n1a2 RECIBIDO: 16 de septiembre de 2015 APROBADO: 20 de enero de 2016

CORRESPONDENCIA:

Armando Luís Vieira, Universidade de Aveiro - DEGEIT GOVCOPP, Campus Universitario de Santiago. 3810-193 Aveiro, Portugal.

a PhD in Business and Management, Assistant Professor, Universidade de Aveiro, Portugal. E-mail: aluisvieira@ua.pt

b PhD in Tourism, Full Professor, Universidade de Aveiro - DEGEIT/GOVCOPP, Portugal. E-mail: ccosta@ua.pt 


\section{Las relaciones comerciales en el campo de la hotelería: un modelo estructural aplicado al segmento corporativo}

RESUMEN El presente artículo tiene como objetivo investigar sobre la influencia de la importancia y la calidad de las relaciones comerciales en la decisión de una empresa de mantener su relación con un determinado hotel. Para abordar el problema, se propone un modelo puesto a prueba con base en los datos de una encuesta de una muestra final de 483 representantes de pequeñas y medianas empresas (PYMEs) de Portugal. Los participantes expresaron sus percepciones acerca de las relaciones comerciales con sus socios clave en una cadena de hoteles. Los datos se analizaron utilizando la técnica estadística de modelos de ecuaciones estructurales. El estudio produjo varios resultados importantes. La calidad de la relación está influenciada por variables interpersonales, entre las cuales se destaca el papel de Administrador de Clientes (es decir, el representante del hotel), y surgen otras variables asociadas a la relación misma. En cuanto a la importancia de la relación, surgen varios factores como indicadores significativos, tales como los beneficios de la relación y los costos de su interrupción. Los resultados sugieren que la importancia de la relación juega un papel preponderante en la determinación de la propensión a la interacción futura. En cuanto a la contribución teórica, la presente investigación añade valor al paradigma del marketing relacional, ya que somete a prueba y valida un modelo de la influencia de la importancia de la relación, además de los efectos de la calidad de la relación en la propensión de la futura interacción entre las PYMEs y los hoteles. Este estudio también sugiere implicaciones prácticas relacionadas con la gestión, a la luz del éxito de las estrategias de marketing relacional, y con el objetivo de fidelizar al cliente y obtener rentabilidad de la relación comercial que se haya establecido.

PALABRAS CLAVE importancia de las relaciones, marketing relacional, modelación en ecuaciones estructurales, cualidades de la relación, relaciones de negocios.

\section{Business relations in hospitality: A structural model applied to the corporate segment}

ABSTRACT This article aims to conduct research on the influence of the importance and quality of business relationships on a company's decision to maintain its relationship with a certain hotel. To address the issue, we propose a model tested based on data from a survey of a final sample of 483 representatives from Portuguese small and medium enterprises (SMEs) who expressed their perceptions about the business relationships with their key partners in a hotel chain. Data were analyzed using the structural equation modeling statistical technique. The study produced several important results. The quality of the relationship is influenced by interpersonal variables, highlighting the role of Client Manager (i.e. the representative of the hotel), and other variables associated to the relationship itself. As for the importance of the relationship, several factors emerge as significant indicators such as the benefits of the relationship and the costs of its interruption. The results suggest that the importance of the relationship plays a major role in determining the propensity for future interaction. As regards theoretical contribution, this research adds value to the relationship marketing paradigm because we test and validate a model of the influence of the importance of the relationship, in addition to the quality of the relationship, and the propensity of future interaction between SMEs and hotels. This study also suggests practical management-related implications, in light of the success of relationship marketing strategies, and with the goal of customer loyalty and profitability of the business relationship which they have established.

KEYWORDS importance of the relationship, relationship marketing, modeling in equations structural quality of the relationship, business relationship. 


\section{Introdução e enquadramento}

A concorrência entre os hotéis em Portugal é cadavezmaior emaisacirrada. Os hotéis interrogam -se sobre a melhor maneira de se manterem competitivos e sobreviverem num mercado limitado como é o das empresas em Portugal. Os hotéis sabem que o sucesso assenta sobre três pilares fundamentais: captação, fidelização e rentabilização de clientes. 0 marketing de um hotel desempenha um papel fundamental no cumprimento desses objetivos, nomeadamente o marketing relacional, que é atualmente um tema de destaque. Segundo a literatura (e.g., Beck et al., 2015; Bradford et al., 2010; Henderson et al., 2011; Krasnikov et al., 2009; Maggon e Chaudhry, 2015; Nyaga e Whipple, 2011; Palmatier et al., 2013), o futuro do marketing passa inevitavelmente pelo marketing relacional. Isso porque o marketing com objetivos imediatistas (hit-and-run marketing) não serve nem aos verdadeiros interesses dos compradores nem aos interesses dos vendedores uma vez que fica mais dispendioso às empresas atraírem novos clientes do que fidelizar os existentes.

Embora, atualmente, a teoria do marketing, por tradição, ainda esteja muito orientada para o produto e para a transação, em lugar do cliente e da relação, existem cada vez mais empresas que adotam estratégias de marketing relacional, designadamente nos serviços hoteleiros em que os clientes realizam elevado número de compras ao longo do tempo. Essa abordagem está em linha com aquilo a que Gümmesson (2014) apelida de "Paradigma 3", que assenta na lógica dominante dos serviços (service-dominant logic) introduzida por Vargo e Lusch (2004) e revisitada recentemente (Lusch e Vargo, 2014), assim como nas teorias de sistemas (Mele et al., 2010) e de relações em rede, nomeadamente em contexto business-to -business (B2B) (Ford et al., 1998; Hakansson et al., 2009). Essas perspetivas realçam a complexidade dos serviços, em que a cocriação de valor nas relações de negócio em contexto de serviços B2B depende fortemente dos contributos de todos os parceiros (ou business partners, se quisermos ser mais abrangentes e usar terminologia anglosaxónica). Gümmesson (2014, p. 660-661) reclama especificamente por mais estudos sobre as realações de negócio na área da hotelaria.

Com efeito, existe hoje uma tendência crescente das empresas para centrarem os seus esforços na fidelização e no aumento da venda cruzada na pequena fatia de clientes rentáveis (a comprovada regra dos 20/80: 20\% dos clientes são responsáveis por $80 \%$ da rendibilidade total). De resto, o fortalecimento de relações com os clientes não é imitável, pelo que constitui um fator de diferenciação e uma vantagem competitiva sustentável. O segmento empresarial (ou B2B ou corporate), também designado por segmento empresas, é um exemplo flagrante de cocriação de valor, de repetição de compra e de rentabilidade (Yelkur e DaCosta, 2001) e isso justifica que seja responsável pela maior fatia do investimento que os prestadores de serviços fazem em esforços de marketing (Gümmesson e Grönroos, 2012). Esses esforços passam em grande medida por uma aposta em interações "actor-to-actor" (Gümmesson, 2014, p. 659), em que cada empresa/organização/instituição é representada por um(a) profissional que é responsável por gerir as relações de negócio com os parceiros na rede de relações em que a sua organização está inserida.

Um sinal evidente da importância da gestão das relações de negócio é a introdução da figura do Gestor de Clientes (ou Promotor Comercial, denominação utilizada em algumas cadeias hoteleiras) e a criação dos Centros de Empresas em alguns hotéis a operar em Portugal, à semelhança do que havia sucedido no setor da banca na década de 1980 (note-se que existem muitas semelhanças entre os dois setores, desde a personalização dos serviços à forte interação cara a cara, passando por aspetos relacionados com a personalidade, o prestígio, a confiança e a segurança). Essa abordagem radica também na ideia de que os compradores obtêm utilidade a partir do capital social (Granovetter, 1985; Hughes, Le Bon e Rapp, 2013).

Todavia, apesar de um consenso generalizado acerca dos benefícios de uma abordagem relacional, gestores e académicos têm vindo a expressar algum desapontamento, porque o marketing relacional produz, por vezes, resultados contraditórios (Palmatier et al., 2009) e poderá não corresponder às expetativas (Henderson, Beck e Palmatier, 2011). Isso poderá acontecer se, por exemplo, for adotada uma abordagem relacional indiscriminada, que não cuida de selecionar criteriosamente os contextos e os segmentos em que o marketing relacional pode ser mais eficaz. Maggon e Chaudhry (2015), com base numa análise de artigos publicados desde o ano de 2001, concluíram que o estudo do marketing relacional na área do turismo e hospitalidade é ainda incipiente e que são necessários mais estudos, nomeadamente 
no que concerne às relações de negócio entre parceiros-chave ("key stakeholders") e à influência dos esforços relacionais na "intenção" de manter e desenvolver as relações de negócio (Maggon e Chaudhry, 2015, p. 64).

Assim, torna-se pertinente estudar os benefícios dos esforços relacionais, designadamente no que concerne à longevidade das relações de negócio em hotelaria no segmento empresarial. Nesse quadro, o enfoque deste trabalho é nas relações de negócio que se estabelecem entre os representantes das organizações envolvidas na relação de negócio, ou seja, no caso concreto, entre o representante da empresa e o seu interlocutor privilegiado no hotel (i.e., o gestor de clientes).

A maior parte da literatura sobre marketing relacional assume implicitamente que o desenvolvimento e manutenção de relações entre vendedores e compradores - neste estudo, entre hotéis e empresas - são objetivos comuns a ambas as partes. Contudo, nem todos os clientes valorizam os benefícios das relações, nem todos lhes atribuem a mesma importância, pelo que os hotéis deverão selecionar cuidadosamente as situações em que o marketing relacional deve ser utilizado (Furash, 1997; Perrien et al., 1993; Vieira, Winklhofer e Ennew, 2014). Com efeito, estudos anteriores demonstraram que a qualidade da relação influencia a propensão de contacto futuro entre comprador e vendedor, o mesmo é dizer, a longevidade das relações entre clientes e hotéis (e.g., Crosby et al., 1990; Boles et al., 2000), mas é necessário averiguar os efeitos da importância da relação na propensão de interação futura, adicionalmente à influência da qualidade da relação.

De facto, se determinada relação não for importante, na perspetiva de uma ou de ambas as partes, a motivação para continuar a interação não será, provavelmente, muito elevada, por muito boa que seja a qualidade da relação. De outro modo, se, para além de ser boa a qualidade, a relação for simultaneamente percebida como importante, é natural que a propensão de interação futura entre a empresa e o hotel seja reforçada pelo efeito da importância da relação. Nesse contexto, a presente investigação tem como objetivo propor e testar um modelo que permita avaliar a influência simultânea da qualidade e da importância das relações de negócio na propensão de interação futura entre hotéis e empresas.

\section{Proposta de modelo}

Tendo em conta a questão para investigação acima descrita, o contexto do presente estudo e um significativo grau de consenso na literatura sobre os constructos que determinam, quer a qualidade da relação, quer a importância da relação (e.g., Crosby, Evans e Cowles, 1990; Vieira, 2001; Palmatier et al., 2006; Vieira, Ennew e Winklhofer, 2008; Athanasopoulou, 2009; Nyaga e Whipple, 2011; Vieira et al., 2014), propõe-se o modelo apresentado na Figura 1.

0 modelo pretende testar se, por um lado, conforme proposto, a componente interpessoal e as características da relação efetivamente determinam a qualidade de uma relação de negócio; por outro lado, avaliar da capacidade dos fatores situacionais e de risco inerente determinarem ou condicionarem a importância de uma relação. Em suma, o modelo pretende averiguar o que influencia a decisão de uma empresa continuar a sua relação de negócio com determinado hotel. As secções seguintes detalham o modelo proposto descrevendo as associações entre as variáveis.

\section{Antecedentes da propensão de interação futura}

\section{Qualidade da relação}

No presente estudo, a qualidade da relação é definida como a avaliação cognitiva conjunta das interações de negócio, por parte de indivíduoschave numa relação B2B (Holmlund, 2001). Em linha com a corrente de opinião predominante na literatura, neste estudo, é adotada a perspetiva de Crosby et al. (1990), que conceptualizaram a qualidade da relação como uma variável que tem como dimensões a confiança e a satisfação. A confiança é definida como a capacidade e a vontade de confiar na integridade e comportamento do gestor de relacionamento, de modo a que as expetativas de longo prazo sejam cumpridas (Crosby et al., 1990; Morgan e Hunt, 1994); a satisfação é definida como a segurança e a tranquilidade percebidas pelo comprador acerca do desempenho futuro do fornecedor, tendo em conta o desempenho satisfatório ocorrido até então (Crosby et al., 1990; Naudé e Buttle, 2000). 
FIGURA 1. Modelo dos efeitos da qualidade e da importância da relação de negócio na propensão de interação futura entre hotel e cliente

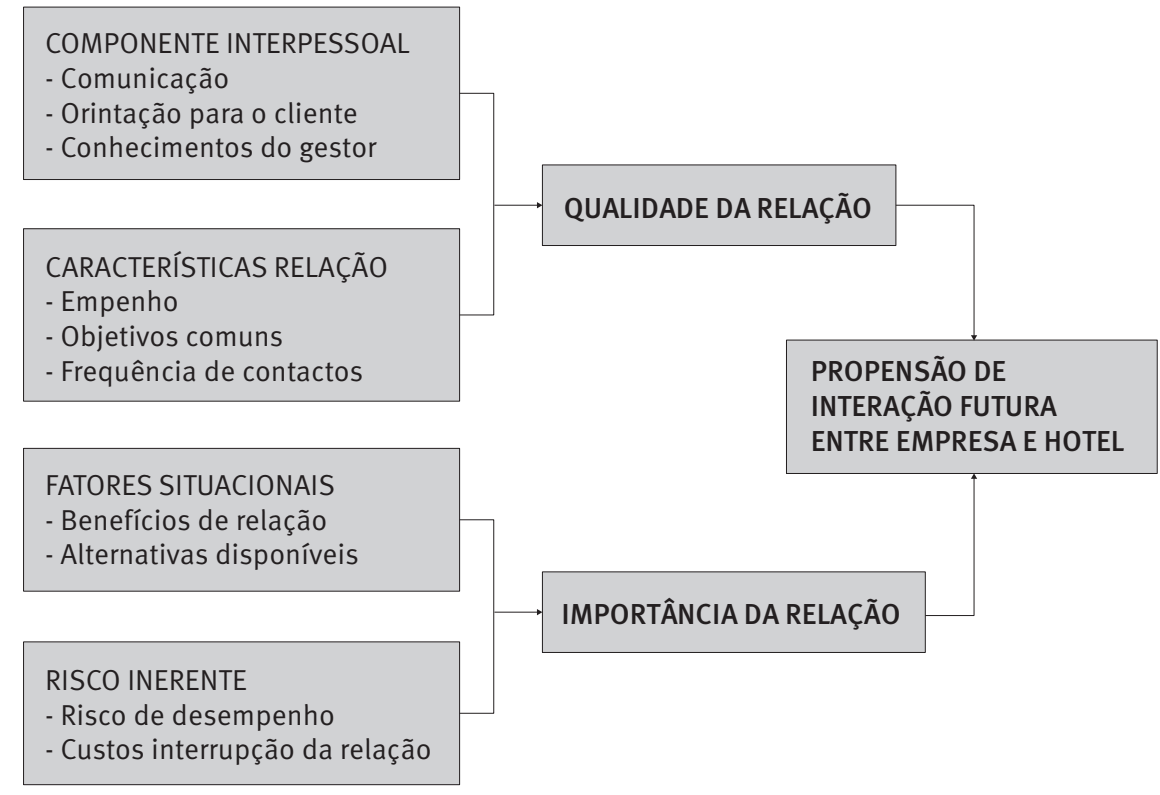

Fonte: elaboração própria.

A propensão de um cliente continuar a fazer negócio com determinado hotel depende, de forma significativa, das expetativas que tem relativamente a esse hotel e da medida em que essas expetativas são satisfeitas. A propensão de interação futura é, portanto, influenciada pela qualidade da relação. Conforme demonstrado por Crosby et al. (1990), a dimensão qualidade da relação exerce um efeito positivo na propensão de interação futura entre as partes, o mesmo é dizer, na propensão de manutenção da relação ou, podemos também dizer, na fidelização dos clientes.

\section{Importância da relação}

Independentemente de a qualidade da relação ser boa ou não, se os serviços de determinado hotel forem cruciais para o desempenho e a sobrevivência da empresa, isto é, se a influência da dimensão importância da relação é elevada, é natural que exista, da parte do cliente, motivação para manter a interação com esse hotel (Frazier, 1983; Vieira et al., 2014; Beck et al., 2015). Desse modo, quanto maior for a importância da relação, maior será a propensão de interação futura entre as partes.

\section{Antecedentes da qualidade da relação}

Comecemos pela componente interpessoal. A comunicação facilita a relação entre as partes e influencia positivamente a perceção que o cliente tem do Gestor de Clientes (Williams et al., 1990), o que sugere que essa variável deverá estar positivamente associada com a qualidade da relação. $\mathrm{Ou}$ seja, quanto mais elevado for o nível das capacidades de comunicação demonstradas pelo Gestor de Clientes, mais elevado será o nível da qualidade da relação. Por outro lado, a comunicação eficaz funciona como determinante da confiança (Morgan e Hunt, 1994) e da satisfação (Leuthesser, 1997).

De acordo com estudos de Saxe e Weitz (1982), a orientação para o cliente está relacionada positivamente com a satisfação e confiança que, conforme referido, são as duas vertentes da qualidade da relação adoptadas por Crosby et al. (1990)—.

O modelo contém ainda a variável conhecimentos do Gestor de Clientes, tendo em conta que os vendedores (o mesmo é dizer, nos hotéis, os Gestores de Clientes) que melhor conhecerem a atividade dos seus clientes são por estes mais valorizados (Perrien e Ricard, 1995). Para além 
disso, os Gestores de Clientes devem ter conhecimentos sólidos sobre a função que desempenham sobre a organização que representam e os seus produtos. De acordo com investigações anteriores (Sujan et al., 1988; Vieira et al., 2008), quanto mais conhecimentos demonstrarem os vendedores, mais confiança inspiram no cliente. Considerando que a confiança é uma das dimensões da qualidade da relação, a variável conhecimentos do Gestor de Cliente deverá estar positivamente relacionada com a qualidade da relação.

Relativamente às características situacionais, uma das variáveis que assumem maior relevo é o empenho. Entende-se por empenho ou compromisso a motivação firme e consistente das partes para manter uma relação que é por elas valorizada (Dwyer et al., 1987; Morgan e Hunt, 1994). Quanto maior for essa motivação, maior será a probabilidade de a qualidade da relação aumentar. Uma relação duradoura está dependente do compromisso mútuo entre o cliente e o prestador de serviços e sugere que a qualidade é essencial para a sua manutenção (Dwyer et al., 1987). De acordo com Lawler e Yoon (1993), o empenho gera a confiança entre as partes e, segundo Molm (1991), o empenho também potencia a satisfação com as relações, o que sugere que o empenho está associado positivamente com a qualidade da relação.

A variável objetivos comuns representa o nível de partilha de objetivos que só são possíveis de atingir por meio da cooperação e da manutenção da relação (Wilson, 1995). Tal como nas relações entre as pessoas, a existência de objetivos comuns traduz-se numa forte motivação para manter a relação, cuja longevidade será tanto maior quanto mais elevado for o grau de concretização desses objetivos e os benefícios dela retirados. Tendo ainda em conta que, de acordo com Wilson (1995), os objetivos comuns podem igualmente potenciar a satisfação cliente com o desempenho do vendedor e com a relação com ele estabelecida, possivelmente também estarão associados com a qualidade da relação.

Em conclusão, no que concerne aos determinantes da qualidade da relação, a frequência de contactos ou visitas dos Gestores de Clientes dos hotéis às empresas que acompanham é (e, nos casos em que não é, devia ser) um dos instrumentos de medida que fazem parte dos inquéritos à satisfação dos clientes. De acordo com Woodside et al. (1992), quanto maior for a frequência dos contactos entre as partes, mais elevada será a probabilidade de sucesso de uma estratégia de relação. Quanto mais frequentes forem os contactos, melhor será a comunicação, o que permitirá ao hotel adequar melhor os seus esforços de marketing às efetivas necessidades dos clientes e, ao mesmo tempo, mais as empresas sentem que os seus problemas também são importantes para o hotel. A frequência dos contactos está também associada ao grau de utilização dos serviços. Assim, é provável que a frequência dos contactos entre as partes seja um indicador da qualidade da relação e que a associação entre os dois fenómenos seja positiva.

\section{Antecedentes da importância da relação}

Para que uma relação perdure e seja considerada importante, as partes têm de sentir que recebem algo por participar nela. A variável benefícios da relação reflete essa realidade no modelo e está incluída nos fatores situacionais. A realização contínua de negócio entre hotéis e empresas gera certos benefícios, intangíveis ou não, económicos ou não, para ambas as partes. Por exemplo, a empresa sente que pode contar com o apoio do hotel e vê amenizado o risco percebido associado aos serviços hoteleiros. 0 hotel, por seu lado, obtém um conhecimento mais aprofundado sobre a empresa, o que constituirá uma vantagem competitiva quando se tratar de decidir se aumenta ou não o seu envolvimento com o cliente (Vieira et al., 2008). Os benefícios de uma relação são, segundo Han et al. (1993), as recompensas decorrentes de participar nessa relação, que não existiriam sem ela. Quanto melhores as recompensas, maior será a importância atribuída pelas partes à relação. Isso sugere que, quanto melhores forem os benefícios percebidos pelas partes, mais importante será a relação.

O comportamento dos clientes nas suas interações com os hotéis pode ser condicionado pela existência de relações alternativas, refletidas no modelo por meio da variável alternativas disponíveis. Muitas vezes, os clientes mantêm-se em determinada relação devido aos investimentos nela realizados e não conseguem tirar partido de melhores relações, eventualmente possíveis com outros fornecedores (Han et al., 1993). Noutros casos, os obstáculos à mudança são ligações económicas ou legais (Holmlund e Kock, 1996). Sempre que as alternativas disponíveis, reais ou percebidas, forem muito limitadas, o cliente 
atribui mais importância à sua actual relação e os seus esforços vão no sentido de a manter (Pardo e Salle, 1994). Pelo contrário, faz sentido inferir que, quanto mais alargado é o leque de alternativas e mais fácil é sair da actual relação, menos importante esta se torna para o cliente.

Quanto à componente risco inerente, o risco de desempenho tem a ver com a incerteza associada à capacidade dos concorrentes do atual fornecedor de serviços corresponderem às expetativas do cliente, em termos de satisfação das suas necessidades. À medida que o risco de desempenho percebido aumenta, o cliente torna-se mais cuidadoso na decisões que toma (Webster, 1993). As boas relações atenuam o risco associado à compra, pelo que a importância da relação deverá ser tanto maior quanto mais elevado for o risco de desempenho percebido pelo cliente.

Finalmente, os custos de interrupção da relação, ou seja, os custos de mudar de hotel, incluem todos os custos esperados de sair de uma relação e os custos de iniciar outra relação. Quanto maior for o investimento efectuado na relação, tanto pelo hotel como pelo cliente, maiores são os custos de mudança percebidos, pelo que aumenta o interesse e a necessidade das partes em manter a relação existente (Morgan e Hunt, 1994). Logo, a relação tenderá a ser tanto mais importante para as partes, quanto maiores forem os custos de mudança.

\section{Metodologia}

A análise foi levada a cabo adotando uma perspetiva de modelação em equações estruturais, com recurso aos softwares LISREL e SPSS, a partir das perceções de 483 representantes de pequenas e médias empresas (PMEs) portuguesas acerca das suas relações de negócio com os seus interlocutores privilegiados nos hotéis. 0 inquérito foi realizado num contexto caracterizado por recorrentes interações entre as partes e o serviço personalizado e cocriado, ou seja, um cenário de pesquisa que se assemelha ao contexto em que o marketing relacional é mais eficaz, como sugerido anteriormente (Palmatier et al., 2006; Vieira, 2013).

A recolha de dados realizada com a colaboração de uma cadeia hoteleira internacional que opera em Portugal, com pontos de venda praticamente em todo o território e com um leque abrangente de clientes empresa, quer em termos de dimensão, quer em termos de diversidade de sector, que foi considerada adequada, tendo em mente a representatividade da amostra.

A maior parte dos respondentes representa PMEs, que empregam menos de 500 pessoas e são representativas da estrutura empresarial em Portugal, que conta com cerca de $98 \%$ de PMEs e apenas $2 \%$ de médias e grandes empresas. Acresce que a distribuição por setores de atividade também está globalmente de acordo com o perfil da economia portuguesa: indústria transformadora, $31 \%$; construção, 23\%; comércio, 33\%; e outros setores (predominantemente serviços), 14\%. A duração das relações de negócio entre as partes está dentro de um intervalo de 6 a 8 anos, o que sugere que empresas e hotéis mantêm relações relativamente longas, apesar de algum grau de rotatividade inerente ao setor hoteleiro. Esse dado relativo à duração das interações de negócio assume relevância acrescida visto que, como sugere Palmatier et al. (2013), a importância relativa dos esforços relacionais pode diferir de acordo com a fase que a relação atravessa.

Todas as medidas incluídas no questionário são baseadas em escalas estabelecidas na literatura, com ligeiras adaptações tendo em conta as especificidades do contexto do presente estudo. Conforme ilustra a Tabela 1, que contém os constructos que compõem o modelo, as escalas utilizadas e respetivas fiabilidades, a variável comunicação é medida por meio do grau de concordância do inquirido quanto a afirmações sobre as capacidades de comunicação do Gestor de Clientes, ou seja, do interlocutor privilegiado da empresa no hotel. Utilizaram-se para a variável comunicação escalas adaptadas do estudo de McQuiston (2001). As variáveis orientação para o cliente e conhecimentos do Gestor de Clientes foram medidas com base em escalas adaptadas de um estudo de Swan et al. (1988). As escalas utilizadas para medir a variável empenho foram adaptadas de escalas desenvolvidas por Morgan e Hunt (1994) e Anderson e Weitz (1992), e a variável objetivos comuns foi avaliada por instrumentos de medida adaptados de escalas desenvolvidas por Kumar et al. (1995).

O número de contactos entre o hotel e o cliente pode ser uma medida da qualidade da relação entre ambos. Quanto mais frequentes forem os contactos entre as partes, mais fácil é o estabelecimento de uma estratégia de relação, melhor essa relação se torna e mais eficaz é a comunicação. A frequência de contactos foi medida utilizando uma 
adaptação de uma escala do inquérito à satisfação dos clientes do Grupo Hoteleiro em análise.

Os benefícios da relação foram medidos utilizando adaptações de escalas de Rusbult (1983) e as alternativas disponíveis foram medidas por meio de escalas adaptadas de Kumar et al. (1995). No que concerne ao risco inerente, o risco de desempenho foi avaliado com base numa escala desenvolvida por Jacoby e Kaplan (1972) e os custos de interrupção da relação foram medidos com base em adaptações de escalas desenvolvidas por Gundlach et al. (1995). Finalmente, para medir a variável qualidade da relação, foram utilizadas adaptações de escalas de Kumar et al. (1995), a importância da relação foi medida por meio de adaptações de escalas da autoria de McQuiston (1989) e de Gundlach et al. (1995), e a propensão de interacção futura entre cliente e hotel foi medida utilizando escalas adaptadas de Kumar et al. (1995).

\section{Discussão dos resultados}

A análise baseada nos resultados do LISREL sugere que o model exibe uma boa qualidade de ajustamento e um poder predictivo relevante (ver Tabela 2).

TABELA 1. Variáveis, escalas e respetiva fiabilidade

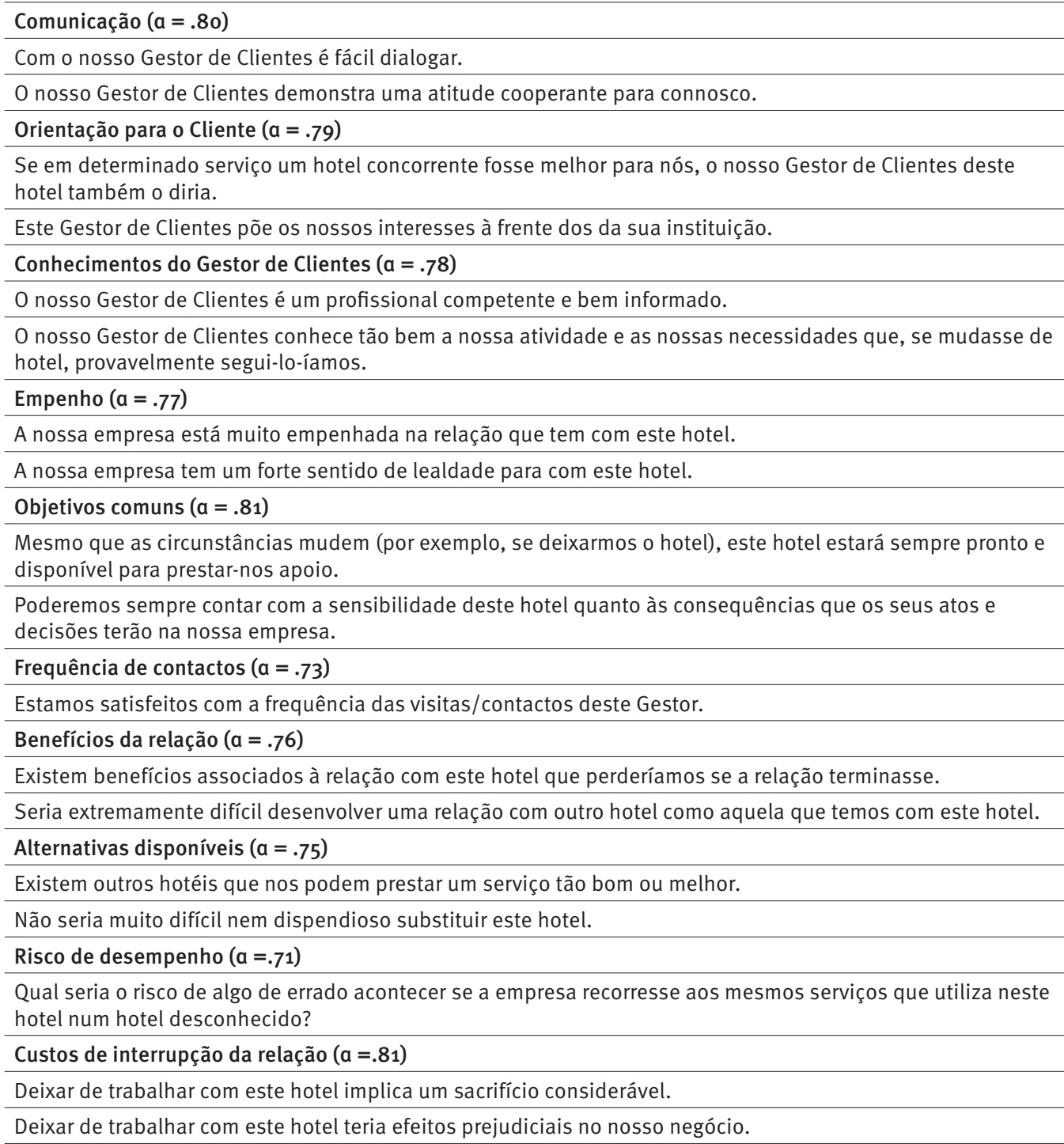


TABELA 2. Coeficientes estandardizados e qualidade do ajustamento

\begin{tabular}{|c|c|}
\hline PROPENSÃO DE INTERAÇÃO FUTURA & $R^{2}=.477$ \\
\hline $\begin{array}{l}\text { Qualidade da relação } \cdots \cdot \text { : propensão } \\
\text { interação futura }\end{array}$ & .322 \\
\hline $\begin{array}{l}\text { Importância da relação } \cdots \cdot \text { ? propensão } \\
\text { interação futura }\end{array}$ & .469 \\
\hline QUALIDADE DA RELAÇÃO & $R^{2}=.453$ \\
\hline Comunicação …: qualidade da relação & .401 \\
\hline $\begin{array}{l}\text { Orientação para o Cliente } \cdots \cdot . \cdot \text { q qualidade } \\
\text { da relação }\end{array}$ & .249 \\
\hline $\begin{array}{l}\text { Conhecimentos do Gestor -.... qualidade } \\
\text { da relação }\end{array}$ & .205 \\
\hline Empenho ..... qualidade da relação & .221 \\
\hline $\begin{array}{l}\text { Objetivos comuns } \cdots . . . * \text { qualidade da } \\
\text { relação }\end{array}$ & .247 \\
\hline $\begin{array}{l}\text { Frequência de contactos } \cdots \cdot . \text { ? qualidade da } \\
\text { relação }\end{array}$ & .267 \\
\hline IMPORTÂNCIA DA RELAÇÃO & $R^{2}=.468$ \\
\hline $\begin{array}{l}\text { Benefícios da relação } \cdots \cdot . \cdot \text { importância da } \\
\text { relação }\end{array}$ & .275 \\
\hline $\begin{array}{l}\text { Alternativas disponíveis .... : importância } \\
\text { da relação }\end{array}$ & .289 \\
\hline $\begin{array}{l}\text { Risco de desempenho -.... importância da } \\
\text { relação }\end{array}$ & $\begin{array}{l}.059 \text { (não } \\
\text { significativa) }\end{array}$ \\
\hline $\begin{array}{l}\text { Custos de interrupção da relação } \cdots \cdot \text {... } \\
\text { importância da relação }\end{array}$ & .269 \\
\hline \multicolumn{2}{|l|}{ ÍNDICES DE AJUSTAMENTO } \\
\hline RMSEA & .054 \\
\hline $\mathrm{CFI}$ & .95 \\
\hline NNFI & .94 \\
\hline$x^{2}$ & 1675.8 \\
\hline
\end{tabular}

Fonte: elaboração própria.

Em termos globais, com a exceção do risco de desempenho, as variáveis propostas como determinantes da qualidade da relação, bem como da importância da relação, revelaram ter uma influência significativa. Quanto à propensão de interação futura, ambos os seus determinantes viram a sua forte influência ser confirmada pelos resultados, com destaque para a importância da relação. É de referir que esses resultados são globalmente mais robustos do que os de uma análise semelhante realizada anteriormente, baseada nas técnicas tradicionais de regressão simples e múltipla (Vieira, 2015), o que vem reforçar a bondade da decisão de utilizar a modelação em equações estruturais para a presente análise. Segue-se uma discussão mais aprofundada dos resultados de cada variável, na qual se estabeleça a ponte com a literatura.

\section{Determinantes da qualidade da relação}

\section{Componente interpessoal}

- Comunicação. A análise sugere a variável comunicação como o mais forte determinante da qualidade da relação, o que está de acordo com resultados de investigações anteriores. Segundo Williams et al. (1990), a comunicação, para além de facilitar a relação, influencia positivamente a perceção do cliente face ao comportamento do Gestor de Clientes. Anderson e Weitz (1989) sugeriram que a comunicação reforça a confiança entre as partes e, portanto, melhora a qualidade da relação. Conforme sugerido por Palmatier et al. (2013) e como será objeto de discussão mais adiante neste trabalho, a circunstância de a variável comunicação, neste estudo, assumir o papel de determinante mais influente poderá ter a ver com a relativa elevada maturidade das relações de negócio identificadas na presente investigação (com duração média num intervalo compreendido entre seis e oito anos).

- Orientação para o Cliente. Os resultados suportam investigações anteriores que concluíram que a orientação para o cliente está associada positivamente com a satisfação e confiança (Saxe e Weitz, 1982) - e, portanto, com a qualidade da relação- e que um serviço orientado para o cliente potencia a satisfação percebida, bem como a qualidade e duração da relação (Kelley, 1992).

- Conhecimentos do Gestor de Clientes. Tal como proposto, o nível de conhecimentos do Gestor de Clientes, percebido pela empresa, está também positiva e significativamente associado com a qualidade da relação. Esses resultados corroboram investigações anteriores na área dos serviços, que sugerem que os clientes valorizam tanto mais os seus interlocutores, quanto mais estes demonstrarem que os conhecem e a sua atividade (Perrien e Ricard, 1995) e que, quanto maior for o nível de conhecimentos demonstrado, maior é a confiança e credibilidade transmitida (Sujan et al., 1988; Vieira et al., 2014), o que potencia a qualidade da relação. 


\section{Características da relação}

- Empenho. O empenho está, igualmente, conforme proposto em hipótese, associado positivamente com a qualidade da relação. Esses resultados corroboram os de investigações anteriores, que indicam que o empenho constitui uma variável determinante no sucesso das relações (Morgan e Hunt, 1994), potencia a confiança entre as partes, uma das vertentes da qualidade da relação (Lawler e Yoon, 1993) e melhora a satisfação, outra das vertentes da qualidade da relação (Molm, 1991). Desse modo, e de acordo com Dwyer et al. (1987), a manutenção de uma relação ao longo do tempo depende da sua qualidade e implica um determinado nível de empenho das partes.

0 facto de a variável empenho assumir um papel relativamente menos importante do que em investigações na mesma área (e.g., Vieira et al., 2014) poderá ter a ver, mais uma vez, com a relativa elevada maturidade das relações de negócio identificadas no presente estudo. Isso é coerente com a sugestão de Palmatier et al. (2013) de que a relevância do empenho cresce até aproximadamente o quarto ano de relação, altura em que começa a decrescer. A secção das conclusões elaborará sobre esses aspetos da investigação.

- Objetivos comuns. Tal como proposto em hipótese, os resultados sugerem que a perceção de que existem objetivos comuns a ambas as partes está positivamente associada à qualidade da relação. De forma semelhante, também Wilson (1995) sugere que a existência de objetivos comuns potenciam a satisfação das partes e constituem forte motivação para manter e fortalecer a relação, na medida da concretização dos objetivos.

- Frequência de contactos. A frequência de contactos tem uma associação positiva com a qualidade da relação, conforme proposto neste estudo e sugerido em investigações anteriores. Com efeito, quanto mais elevada for a frequência de contactos entre as partes, tanto maior será a propensão de sucesso de uma estratégia de relação (Woodside et al., 1992). Na perspetiva inversa, tal como concluiu Smith (1989), quanto menos frequentes forem os contactos, maior é a possibilidade de o cliente pensar que o hotel não está interessado nele, o que não abona nada em favor da qualidade da relação.

\section{Determinantes da importância da relação}

\section{Características da situação}

- Benefícios da relação. Os resultados validam a hipótese formulada, que propunha uma associação positiva entre os benefícios percebidos pelas partes e a importância da relação. São também compatíveis com a revisão de literatura efectuada. Recordando brevemente, os benefícios são as recompensas decorrentes de participar em determinada relação (Han et al., 1993). Quanto melhores forem as recompensas obtidas (ou esperadas), mais importante a relação e maior é a motivação para a manter (Morgan e Hunt, 1994). Desse modo, os clientes parecem estar dispostos a manter as relações cujo rácio entre os benefícios e os sacrifícios percebidos, usando a terminologia de Monroe (1991), lhes seja favorável e mostram-se disponíveis para mudar quando esse rácio é melhor noutro hotel.

- Alternativas existentes. Os resultados corroboram a ideia de que, quanto mais pobre for o número de alternativas, reais ou percebidas, à disposição do cliente, mais importante se torna a relação. Essa situação pode ocorrer por vários motivos, por exemplo, devido aos investimentos já efetuados na relação actual (Han et al., 1993) ou a ligações económicas ou legais (Holmlund e Kock, 1996). Inversamente, quando o leque de alternativas é alargado, como é o caso da conjuntura actual da hotelaria em Portugal, os clientes assumem uma posição de maior exigência face ao comportamento que esperam dos hotéis. Digamos que, se o número de hotéis alternativos à disposição do cliente for elevado, a relação torna-se menos importante, até porque, provavelmente, o cliente passa a deter uma certa superioridade na relação, um certo controlo sobre a outra parte. Uma forma de os hotéis combaterem essa dificuldade poderá ser especializarem-se em áreas particularmente sensíveis a determinados setores empresariais.

\section{Factores de risco inerente}

- Risco de desempenho. Contrariamente à hipótese formulada, a influência da variável risco de desempenho revelou ser estatisticamente não 
significativa. A expetativa era a de que os resultados corroborassem a ideia de que quanto maior é o risco de desempenho percecionado pelo cliente, maior é importância atribuída à relação. Tal como sugeriu Webster (1993), à medida que o risco de desempenho aumenta, mais cuidadoso o cliente se torna nas decisões e atribui mais importância à relação em que está envolvido. Assim, quando a perceção é de que o risco de um eventual desempenho insatisfatório de um concorrente é elevado, os clientes podem não optar por mudar de hotel, mesmo que não estejam satisfeitos com a relação, porque a mudança poderia eventualmente acarretar ainda mais transtorno à empresa. No caso presente, a análise não corroborou essa linha de pensamento, provavelmente porque as diferenças entre os prestadores de serviços, em termos de nível de qualidade e fiabilidade na prestação de serviços, não justificam os receios de que um hotel concorrente não conseguisse corresponder satisfatoriamente às necessidades da empresa.

- Custos de interrupção da relação. Os resultados sugerem que a variável custos de interrupção da relação funciona como indicador significativo da importância da relação. De acordo com Morgan e Hunt (1994), os custos de interrupção são a combinação dos custos de terminar uma relação com os custos de iniciar uma outra. Quanto maiores forem esses custos, em termos de perceção do cliente, mais importante se torna a relação. Isso pode acontecer devido a vários fatores. Ầ medida que aumentam os investimentos na construção da relação -e enquanto estes não forem recuperados pelas partes-, elevam-se também os custos de mudança e aumenta o interesse em manter a relação (Morgan e Hunt, 1994). Quanto maior for a interdependência entre as partes, mais dispendioso se torna interromper a relação, e sobrepõem-se a cooperação e o empenho ao conflito e à rutura (Pardo e Salle, 1994). Por outro lado, quanto mais antiga for a relação, maior é o conhecimento e a confiança entre as partes, pelo que os custos de interrupção aumentam com a antiguidade da relação (Anderson e Weitz, 1989).

\section{Propensão de interação futura e seus determinantes}

Tanto a qualidade da relação como a importância da relação, face aos resultados obtidos no presente estudo, constituem-se como determinantes significativos da probabillidade de interação futura entre a empresa e o hotel. Os resultados suportam as associações propostas. Conclui-se, assim, que, à medida que aumenta a qualidade percebida, mais provável se torna a manutenção da relação, o que está de acordo com resultados de investigações anteriores, nomeadamente de Crosby et al. (1990).

Todavia, e tendo em conta a globalidade dos resultados, a qualidade da relação parece ser uma condição necessária à continuidade da interação, mas não suficiente. Esse ponto da discussão tem a ver especificamente com o objetivo traçado para a presente investigação: avaliar dos efeitos da importância da relação na comprovada influência que a qualidade da relação exerce sobre a propensão de interação futura entre empresas e hotéis. De facto, a análise aponta a importância da relação como forte determinante da propensão de interação futura, conforme havia sido sugerido inicialmente por Frazier (1983) e, no caso presente, os resultados indicam que a influência da importância da relação se sobrepõe à da qualidade da relação quando a empresa toma a decisão de manter ou não a interação com determinado hotel.

\section{Conclusões}

A presente investigação visa acrescentar valor ao paradigma do marketing relacional desenvolvendo um modelo ou estrutura conceptual que possa constituir mais uma contribuição para a compreensão da problemática das relações de negócio entre empresas e hotéis. Com efeito, a presente investigação proporciona importantes contribuições teóricas na medida em que propõe e valida, por meio de um processo robusto e rigoroso de análise, um modelo ilustrativo da influência da importância da relação, conjuntamente com a qualidade da relação, na decisão de uma empresa em manter e reforçar o envolvimento com determinado hotel. 0 modelo considera também a influência dos determinantes, quer da qualidade da relação, quer da importância da relação, pelo que se espera que constitua uma base de trabalho útil e um estímulo para futuras investigações.

Se atentarmos nas associações entre os constructos que compõem o modelo (rever Figura 1), constatamos que o estudo revela que as relações de qualidade elevada dependem: i) de factores interpessoais, tais como as capacidades de comunicação, de orientação para o cliente e o nível 
de conhecimentos do Gestor de Clientes; ii) de características da relação, tais como o empenho das partes, a existência de objetivos comuns e da frequência de contactos entre a empresa e o hotel. Frequentemente, surge a questão de se saber se as empresas estabelecem relações com as pessoas ou com as organizações por elas representadas. 0 estudo sugere que, embora ambas as vertentes sejam importantes e as relações de negócio sejam interações institucionais, a vertente interpessoal, o "cara a cara" (o actor-to-actor) entre o Gestor de Clientes e o seu interlocutor privilegiado na empresa desempenha um papel crucial em todo o processo.

O presente estudo também sugere que a influência da importância da relação é preponderante. A análise levada a cabo identifica vários fatores que determinam a importância de uma relação. As relações assumem maior importância nas situações que proporcionarem às partes o maior número de benefícios e em que o número de alternativas disponíveis for reduzido (que parece não ser o caso da hotelaria portuguesa, na conjuntura actual, tendo também em conta a ausência de especialização da hotelaria) —são os fatores situacionais-. A importância das relações também depende dos custos de interrupção, que correspondem aos custos de interromper uma relação adicionados aos custos de iniciar uma relação alternativa (os hotéis estão particularmente atentos a esse aspecto dedicando especial atenção à venda cruzada).

Em termos de implicações práticas de gestão empresarial, espera-se que os resultados da investigação constituam um contributo para que os hotéis conheçam melhor os fatores que são mais importantes na relação entre as PME e a hotelaria, uma vez que o estudo tem em conta a perspetiva deste segmento da sua clientela, no sentido de melhor adequarem a sua oferta às efectivas necessidades dos clientes. Espera-se igualmente contribuir para o sucesso da implementação de estratégias de marketing relacional na hotelaria, nomeadamente com vista à fidelização dos clientes e à rentabilização das relações com eles estabelecidas.

Por exemplo, é necessário ter em conta que o Gestor de Clientes (o mesmo é dizer, o interlocutor privilegiado do cliente no hotel) desempenha um papel crucial para o sucesso das relações. Desse modo, os hotéis devem conceder especial atenção à seleção, formação e motivação dos colaboradores que representam o hotel junto dos clientes, bem como a factores perturbadores das relações, tais como a elevada rotatividade dos Gestores de Clientes e a sua falta de competências de decisão. Tendo em conta que existe cada vez menos espaço para a competitividade via oferta e preço, desenvolver relações boas e duradouras com os clientes afigura-se como uma forma de vantagem competitiva sustentável. De facto, as ligações baseadas em bens e/ou serviços, face ao seu cada vez mais curto ciclo de vida, podem ser, para além de imitáveis, apenas esporádicas, o que não permitiria a sua rentabilização adequada. Provavelmente, nem todos os clientes valorizam os benefícios das relações. Desse modo, o marketing relacional deve ser entendido apenas como mais um instrumento eficaz à disposição das organizações, nesse caso dos hotéis, para rentabilizar as relações estabelecidas com os clientes.

Nesse contexto, outra contribuição do presente estudo para melhorar o desempenho de quem está no terreno no dia a dia é alertar para o risco de se fazerem investimentos indiscriminados em esforços relacionais, dado que podem ser altamente ineficazes e contraproducentes. Por um lado, é necessário ter em mente que a importância relativa dos esforços relacionais pode diferir de acordo com a fase que a relação de negócio atravessa Palmatier et al. (2013). Essa constatação sugere que os gestores no terreno podem tirar mais partido do marketing relacional se apostarem nos esforços relacionais que, em cada momento, podem gerar melhores resultados. Por exemplo, a confiança poderá ser mais importante no início da relação, em que as partes ainda estão a conhecer-se (Dwyer et al., 1987). À medida que a relação avança e entra na rotina, a confiança entre as partes pode tornar-se apenas um requisito mínimo e outras variáveis, como, por exemplo no caso concreto do presente estudo, a comunicação e a orientação para o cliente podem assumir um papel preponderante. Essa ideia está em linha com Palmatier et al. (2013), que concluíram que a importância do empenho tem uma trajectória crescente até a relação completar quatro anos e depois começa a decrescer.

0 facto de, no caso da presente investigação, a duração média das relações entre os representantes das partes se situar entre os seis e os oito anos é coerente com a preponderância da variável comunicação relativamente, por exemplo, a variáveis como o empenho e os conhecimentos do gestor. Efetivamente, quando uma relação entra numa fase mais rotineira, as capacidades de 
comunicação assumem um papel preponderante, por exemplo, para convencer o cliente de que ambas as partes continuam empenhadas e a partilhar objetivos. Isso pode ser conseguido investindo tempo em entender onde estão as oportunidades de o fornecedor de serviços ir ao encontro das necessidades do negócio do cliente, considerando as ofertas da concorrência.

Por outro lado, os gestores têm de ter consciência de que uma abordagem relacional não pode ser encarada como uma solução para todos os problemas (one-size-fits-all). Pelo contrário, os esforços relacionais devem ser direcionados para os segmentos em que é possível obter melhores resultados como, por exemplo, o segmento empresarial, no qual é possível existirem contactos personalizados e frequentes -e, logo, a cocreação de valor - entre os representantes das partes (o que é relativamente mais difícil de conseguir no segmento dos clientes individuais). Nesse contexto, como sugerem Bradford et al. (2010), será possível atingir uma integração da força de vendas do fornecedor do serviço com a equipa do cliente, em que ambas as partes trabalham, não apenas com os seus departamentos de marketing e outras áreas funcionais internas, mas também de forma sincronizada com os parceiros, como se tratasse de entidade una e comum a ambas as organizações, a vendedora e a compradora.

\section{Limitações e sugestões para investigações futuras}

Os resultados deste estudo devem ser vistos num contexto com certas limitações que podem, simultaneamente, abrir novas avenidas de investigação. Por exemplo, aos inquiridos foi solicitada a descrição das suas relações com apenas um fornecedor e, portanto, não foi possível avaliar se as demais interações que se estabelecem na rede de relações em que cada empresa está envolvida, que inclui várias cadeias hoteleiras, influenciam a relação em análise e em que medida. Por outro lado, o presente estudo centrou-se na perspetiva dos clientes. Impõe-se, portanto, auscultar a opinião dos responsáveis dos hotéis quanto aos efeitos da qualidade da relação e a da importância da relação na propensão de interação futura entre as partes.

Acresce que, embora as variáveis incluídas na estrutura conceptual proposta se tenham confirmado como indicadores significativos, existem provavelmente outras variáveis que poderão acrescentar poder explicativo ao modelo. Uma das variáveis que tem sido sugerida como merecedora de estudos mais aprofundados é o poder negocial que, em caso de assimetria, poderá ter efeitos contraproducentes (Beritelli e Laesser, 2011; Guo e He, 2012; Nyaga et al., 2013). 0 assunto em estudo é, de facto, marcadamente multifacetado. Sugerem-se, pois, investigações futuras, por exemplo, no domínio das novas tecnologias de informação e comunicação e das respectivas implicações no conceito de relacionamento hoteleiro e no papel que nele têm as relações interpessoais; além disso, no domínio do marketing interno, tendo em conta que os serviços hoteleiros são altamente intensivos em contactos pessoais e que a função desempenhada pelos agentes comerciais que asseguram a relação cara a cara com os clientes é crucial para o marketing relacional.

\section{REFERÊNCIAS}

Athanasopoulou, P. (2009). Relationship quality: A critical literature review and research agenda. European Journal of Marketing, 43(5/6), 583-610.

Anderson, E. e Weitz, B. (1989). Determinants of Continuity in Conventional Industrial Channel Dyads. Marketing Science, 8(outono), 310-23.

Anderson, E. e Weitz, B. (1992). The Use of Pledges to Build and Sustain Commitment in Distribution Cannels. Journal of Marketing Research, 29(fev.), 18-34.

Beck, J. T., Chapman, K. e Palmatier R. W. (2015). Understanding Relationship Marketing and Loyalty Program Effectiveness in Global Markets. Journal of International Marketing, 23(3), 1-21.

Beritelli, P. e Laesser, C. (2011). Power Dimensions and Influence Reputation in Tourist Destinations: Empirical Evidence from a Network and Stakeholders. Tourism Management, 32, 1299-1309.

Boles, J. S., Johnson, J. e Barksdale, H. C. (2000). How Salespeople Build Quality Relationships: A Replication and Extension. Journal of Business Research, 48, 75-81.

Bradford, K., S. Brown, S. Ganesan, G. Hunter, V. Onyemah, R., Palmatier, D. Rouziès, R. Spiro, H. Sujan e Weitz, B. (2010). The embedded sales force: Connecting buying and selling organizations. Marketing Letters, 21, 239-53.

Crosby, L. A., Evans, K. E. e Cowles, D. (1990). Relationship Quality in Services Selling: An Interpersonal Influence Perspective. Journal of Marketing, 54(jul.), 68-81. 
Dwyer, F.R., Schurr, P. H. e Oh, S. (1987). Developing Buyer-Seller Relationships. Journal of Marketing, 51(abril), 11-27.

Evans, J. R. e Laskin, R. L. (1994). The Relationship Marketing Process: A Conceptualization and Application. Industrial Marketing Management, 23, 438-52.

Ford, D., Gadde, L.-E., Hakanson, H., Lundgren, A., Snehota, I., Turnbull, P. et al. (1998). Managing Business Relationships. Chichester: John Wiley \& Sons.

Frazier, G. L. (1983). Interorganizational Exchange Behaviour in Marketing Channels: A Broadened Perspective. Journal of Marketing, 47(outono), 68-78.

Furash, E. E. (1997). Management Strategies: What's in it for me? Journal of Lending \& Credit Risk Management, 79(ago.), 7-12.

Granovetter, M. (1985). Economic action and social structure: The problem of embeddedness. American Journal of Sociology, 91, 481-510.

Gümmesson, E., (2014). Productivity, quality and relationship marketing in service operations. International Journal of Contemporary Hospitality Management, 26(5), 656-662.

Gümmesson, E. e Grönroos, C. (2012). The emergence of the new service marketing: Nordic school perspectives. Journal of Service Management, 23(4), 479-497.

Gundlach, G., Achrol, R. e Mentzer, J. (1995). The Structure of Commitment in Exchange. Journal of Marketing, 59 (jan.), 78-92.

Guo, X. e He, L. (2012). Tourism supply-chain coordination: the cooperation between tourism hotel and tour operator. Tourism Economics, 18(6), 13611376.

Hakansson, H., Ford, D., Gadde, L.-E., Snehota, I. e Waluszewsky, A. (2009). Business in Networks. Chichester: Wiley.

Han, S. L., Wilson, D. T. e Dant, S. P. (1993). Buyer-Supplier Relationships Today. Industrial Marketing Management, 22, 331-338.

Henderson, C. M., Beck, J. T. e Palmatier, R. W. (2011). Review of the theoretical underpinnings of loyalty programs. Journal of Consumer Psychology, 21(3), 256-276

Holmlund, M. (2001). The D\&D model: Dimensions and domain of relationship quality perceptions. Service Industries Journal 1(3), 13-36.

Holmlund, M. e Kock, S. (1996). Relationship Marketing - The Importance of Customer-Perceived Service Quality In Retail Banking. Service Industries Journal, 16(3), 287-304.

Hughes, D. E., Le Bon, J. e Rapp, A. (2013). Gaining and leveraging customer-based competitive intelligence: The pivotal role of social capital and salesperson adaptive selling skills. Journal of the Academy of Marketing Science, 41, 91-110.

Jacoby, J. e Kaplan, L. B. (1972). The Components of Perceived Risk. Em M. Venkatesan (Ed.), SV Proceedings of the Third Annual Conference of the Association for Consumer Research (382-393). Chicago, IL: Association for Consumer Research.

Kelley, S. W. (1992). Developing Customer Orientation Among Service Employees. Journal of the Academy of Marketing Science, 20 (inverno), 27-36.

Krasnikov, A., Jayachandran, S. e Kumar, V. (2009). The impact of customer relationship management implementation on cost and profit efficiencies: Evidence from the U.S. commercial banking industry. Journal of Marketing, 73(6), 61.

Kohli, A. K. (2011). From the editor: Reflections on the Review Process. Journal of Marketing 75(nov.), 1-4.

Kumar, N., Scheer, L. K. e Steenkamp, J. B. (1995). The Effects of Perceived Interdependence on Dealer Attitudes. Journal of Marketing Research, 32(ago.), 348-356.

Lawler, E. J. e Yoon, J. (1993). Power and the Emergence of Commitment Behavior in Negotiated Exchange. American Sociological Review, 58(ago.), 465-481.

Leuthesser, L. (1997). Supplier relational behaviour: An empirical assessment. Industrial Marketing Management, 26, 245-54.

Lusch, R. F. e Vargo, S. L. (2014). Service-Dominant Logic: Premises, Perspectives, Possibilities. Nova York: Cambridge University Press.

Maggon, M. e Chaudhry, H. (2015). Revisiting Relationship Marketing and Customer Relationship Management in Leading Tourism and Hospitality Journals: Research Trends from 2001 to 2013. Journal of Relationship Marketing, 14(1), 53-77.

Mele, C.; Pels, J. e Polese, F. (2010). A brief review of systems theories and their managerial applications. Service Science, 2(1/2), 126-135.

McQuiston, D. H. (1989). Novelty, Complexity and Importance as Casual Determinants of Industrial Buyer Behavior. Journal of Marketing, 53(abril), 66-79.

McQuiston, D. H. (2001). A Conceptual Model for Building and Maintaining Relationships between Manufacturers' Representatives and Their Principals. Industrial Marketing Management, 30, 165-181.

Molm, L. (1991). Affect and Social Exchange: Satisfaction in Power-Dependence Relationships. American Sociological Review, 56, 475-493.

Monroe, K. B. (1991). Pricing - Making Profitable Decisions. Nova York, McGraw-Hill.

Morgan, R. M. e Hunt, S. D. (1994). The Commitment Trust Theory of Relationship Marketing. Journal of Marketing, 58(jul.), 20-38.

MORIARTY, R.T. e Kimball, R. C. e Gay, J. H. (1983). The management of corporate banking 
relationships. MIT Sloan Management Review, 24(3), 3-16.

Naudé, P. e Buttle, F. (2000). Assessing Relationship Quality. Industrial Marketing Management, 29, 351-61.

Nyaga, G., Lynch, D, Marshall, D. e Ambrose, E. (2013). Power asymmetry, adaptation and collaboration in dyadic relationships involving a powerful partner. Journal of Supply Chain Management, 49(3), 42-65.

Nyaga, G. N. e Whipple, J. M. (2011). Relationship Quality and Performance Outcomes: Achieving a Sustainable Competitive Advantage. Journal of Business Logistics, 32(4), 345-360.

Palmatier, R. W., Houston, M. B., Dant, R. P. e Grewal, D. (2013). Relationship Velocity: Toward a Theory of Relationship Dynamics. Journal of Marketing 77(jan.): 13-30.

Palmatier, R. W., Jarvis, C. B., Bechkoff, J. R. e Kardes, F. R. (2009). The role of customer gratitude in relationship marketing. Journal of Marketing, 73, 1-18.

Palmatier, R. W., Dant, R. P., Grewal, D. e Evans, K. R. (2006). Factors Influencing the Effectiveness of Relationship Marketing: A Meta-Analysis. Journal of Marketing, 70(4), 136-153.

Pardo, C. e Salle, R. (1994). Strategic Interplays of an Actor in a Relationship with a Distributor. Industrial Marketing Management, 23, 403-18.

Perrien, J., Filiatraut, P. e Ricard, L. (1993). The Implementation of Relationship Marketing in Commercial Banking. Industrial Marketing Management, 22, 141-148.

Perrien, J. e Ricard, L. (1995). The Meaning of a Marketing Relationship: A Pilot Study. Industrial Marketing Management, 24(1), 37-43.

Rusbult, C. E. (1983). A Longitudinal Test of the Investment Model: The Development (and Deterioration) of Satisfaction and Commitment in Heterosexual Involvemnets. Journal of Personality and Social Psychology, 45(jul.), 101-17.

Saxe, R. e Weitz, B. A. (1982). The Soco Scale: A Mesure of the Customer Orientation of Salespeople. Journal of Marketing Research, 19(ago.), 343-51.

Smith, A. M. (1989). Service Quality: Relationships Between Banks and Their Small Business Clients. International Journal of Bank Marketing (5), 28-35.
Sujan, H., Weitz, B. A. e Sujan, M. (1988). Increasinng Sales Productivity by Getting Salespeople to Work Smarter. Journal of Personal Selling and Sales Management, 8(ago.), 9-19.

Swan, J. E., Trawick, F., Rink, D. R. e Roberts, J. J. (1988). Measuring Dimensions of Purchaser Trust of Industrial Salespeople. Journal of Personal Selling and Sales Management, 8(maio), 1-9.

Vargo, S. e Lush, R. (2004). Evolving to a New Dominant Logic for Marketing. Journal of Marketing, 68(jan.), 1-17.

Vieira, A. L. (2013). Assessing Relationship Quality and its Key Constructs from a Rival Models Approach. Management Research, 11(2), 113-132.

Vieira, A. L. (2015). Relações de Negócio: os serviços hoteleiros e o segmento PME. Faro: Sílabas e Desafios.

Vieira, A. L., Winklhofer, H. e Ennew, C. T. (2008). Relationship Quality: Literature Review and Research Agenda. Journal of Customer Behaviour, 7(4), 269-291.

Vieira, A. L., Winklhofer, H. e Ennew, C. T. (2014). The Effects of Relationship Marketing on Share of Business: A Synthesis and Comparison of Models. Journal of Business-to-Business Marketing, 21, 1-24.

Webster, C. (1993). Buyer Involvement in Purchasing Success. Industrial Marketing Management, 22, 199-205.

Williams, K. C. e Spiro, R. L. (1985). Communication Style in the Salesperson-Customer Dyad. Journal of Marketing Research, 22(nov.), 434-442.

Williams, K. C., Spiro, R. L. e Fine, L. M. (1990). The Customer-Salesperson Dyad: An Interaction/Communication Model and Review. Journal of Personal Selling and Sales Management, 10 (verão), 29-43.

Wilson, D. T. (1995). An Integrated Model of Buyer-Seller Relationships. Journal of the Academy of Marketing Science, 2(4), 335-345.

Woodside, A. G., Wilson, E. J. e Milner, P. (1992). Buying and Marketing CPA Services. Industrial Marketing Management, 21, 265-272.

Yelkur, R., DaCosta, M. (2001). Differential Pricing and Segmentation on the Internet: The Case of Hotels. Management Decision, 39(4), 252-261. 